\title{
The phospholipid fraction obtained from egg yolk reduces blood pressure increase induced by acute stress in spontaneously hypertensive rats
}

\author{
*Helena Martynowicz ${ }^{1, C, D},{ }^{*}$ Dorian Nowacki ${ }^{1, A-C}$, Grzegorz Mazur ${ }^{1, E, F}$, Tadeusz Trziszka ${ }^{2, A, F}$, Andrzej Szuba ${ }^{3, A, E, F}$ \\ ${ }^{1}$ Department and Clinic of Internal and Occupational Diseases and Hypertension, Faculty of Medicine, Wroclaw Medical University, Poland \\ ${ }^{2}$ Department of Animal Products Technology and Quality Management, Wrocław University of Environmental and Life Sciences, Poland \\ ${ }^{3}$ Division of Angiology, Faculty of Health Sciences, Wroclaw Medical University, Poland \\ A - research concept and design; B - collection and/or assembly of data; C - data analysis and interpretation; \\ $\mathrm{D}$ - writing the article; $\mathrm{E}$ - critical revision of the article; $\mathrm{F}$ - final approval of the article
}

Address for correspondence

Dorian Nowacki

E-mail:dorian.nowacki@wp.pl

\section{Funding sources}

The study was conducted within the project "Innovative Technologies of Production of Biopreparations Based on New Generation Eggs", Innovative Economy Operational Program Priority 1.3.1, thematic area "Bio". The project was co-financed by the European Union through the European Regional Development Fund within the Innovative Economy Operational Program, 2007-2013.

Conflict of interest

None declared

* Helena Martynowicz and Dorian Nowacki contributed equally to this work.

Received on October 24, 2016

Reviewed on February 3, 2017

Accepted on 0ctober 8, 2018

\begin{abstract}
Background. Despite continuous research, an improved understanding of the pathophysiology of hypertension and the development of new antihypertensive therapies, hypertension is still the most prevalent chronic cardiovascular disease (CVD) among adults in Western societies. Stress is a common factor related to cardiovascular morbidity. An increase in blood pressure is one of the most common reactions to stress. Chronic and acute stress have also been related to cardiovascular disorders. Polyunsaturated fatty acids (PUFAs) have attracted considerable interest as potential complementary therapy for the treatment of CVD, including hypertension. Egg yolk, rich in PUFAs and phospholipids, might be a good source of phospholipids and PUFAs.

Objectives. The current study was aimed at investigating the potential effect of the phospholipid fraction of eggs on blood pressure changes in spontaneously hypertensive rats (SHRs) as a response to acute stress.

Material and methods. Male, 7-week-old SHRs received dietary phospholipid fraction for 12 weeks. The control animals received standard feed. At the end of the treatment, they were exposed to 40 min of white noise in order to induce acute stress. Then, blood pressure measurements were carried out under normal conditions for $12 \mathrm{~h}$ before and during the acute stress procedure. Blood pressure was measured using telemetry.

Results. Diastolic, systolic and mean blood pressure were significantly lower in the SHRs treated with phospholipid fraction than in the control group. The acute stressor caused a significant increase in diastolic, systolic and mean blood pressure in both the treated and untreated animals, but the increase in blood pressure as a response to the stressor was significantly less pronounced in the rats treated with phospholipid fraction than in control rats. These results show that egg phospholipids can limit a stress-induced rise in blood pressure.

Conclusions. The results of this research show that phospholipid fraction derived from egg yolk reduces stress-induced increases in blood pressure.
\end{abstract}

Key words: stress, hypertension, phospholipid fraction, hen eggs

DOI

10.17219/acem/97377

\section{Copyright}

Copyright by Author(s)

This is an article distributed under the terms of the

Creative Commons Attribution Non-Commercial License

(http://creativecommons.org/licenses/by-nc-nd/4.0/) 


\section{Introduction}

Eggs are an inexpensive and low-calorie food source which contains many substances indispensable for the development of a young organism, including minerals, proteins, n-3 polyunsaturated fatty acids (PUFAs), and phospholipids. However, eggs are also a source of cholesterol; therefore, the relationship between egg consumption and the risk of cardiovascular disease (CVD) remains controversial. Recently, the remarkable role of PUFAs in the prevention and treatment of CVD, including hypertension, has been described. The consumption of n-3 PUFAs reduces heart rate and systolic and diastolic blood pressure. ${ }^{1,2}$ The consumption of freshwater fish (300-600 g daily) is associated with increased plasma concentrations of n-3 PUFAs, lower blood pressure and lower plasma lipid concentrations. ${ }^{3}$ A metaanalysis of the effect of n-3 PUFAs in fish oil on blood pressure concluded that a higher consumption of n-3 PUFAs was associated with a higher reduction in blood pressure, particularly in hypertensive subjects and those with lipid disorders and atherosclerosis. ${ }^{4}$ Also, n-3 PUFA consumption improves endothelial function. ${ }^{5}$ The reduction in blood pressure may result from increased nitric oxide production, a reduced vasoconstrictive response to catecholamines and angiotensin I, improved vasodilatory responses, arterial compliance, ${ }^{6,7}$ and reduced oxidative stress.

Hypertension is the most common chronic CVD in adults ${ }^{8,9}$ and according to the WHO, it is the leading cause of death worldwide. ${ }^{10,11}$ Known hypertension risk factors include age, gender, obesity, lifestyle (diet and physical activity), ${ }^{12,13}$ and additional cardiovascular risk factors (e.g., diabetes, dyslipidemia or insulin resistance). ${ }^{14,15}$ Chronic stress is linked to all cardiovascular disorders, including hypertension. Through the repeated occurrence of acute stressors and the activation of physiological stress-mediating systems, including the autonomic system, hypothalamicpituitary-adrenal axis and immune system, chronic stress causes an elevation in circulating catecholamine and cortisol levels. Circulating catecholamines acutely elevate blood pressure and contribute to vascular stiffening and cardiac hypertrophy. ${ }^{16}$ A stimulation of adrenergic activity increases sodium resorption, promoting blood pressure elevation. ${ }^{17}$ Elevated levels of cortisol additionally activate the reninangiotensin-aldosterone system, contribute to insulin resistance and hyperinsulinemia, and suppress nitric oxide, kallikrein and prostacyclin production.

Despite the progress in pharmacotherapy, hypertension treatment is still insufficient and the majority of patients worldwide have inadequately controlled blood pressure. ${ }^{18}$ Although antihypertensive drugs play a fundamental role in blood pressure management, they may cause side effects; therefore, a number of studies have searched for food substances that can help reduce or prevent hypertension. In recent years, functional foods have attracted considerable interest as potential complementary therapies for the treatment of hypertension. The aim of this study was to evaluate the impact of a diet enriched with phospholipid fraction obtained from hen egg yolk on blood pressure in spontaneously hypertensive rats (SHRs) exposed to acute stress.

The study was conducted within the project "Innovative Production Technologies of Biopreparation Based on New Generation Eggs (OVOCURA)". We report here the primary findings of OVOCURA.

\section{Material and methods}

Male SHRs were purchased from Charles River Laboratories (Hamburg, Germany) and maintained under specific pathogen-free conditions in a temperature-controlled room $\left(22 \pm 2^{\circ} \mathrm{C}\right)$ with a 12-hour light/dark cycle. All rats had ad libitum access to food and drinking water throughout the experiments. The experiments on animals were performed in accordance with relevant guidelines and regulations, and were conducted with the approval of the Local Ethical Commission at the Institute of Immunology and Experimental Therapy of the Polish Academy of Sciences in Wrocław, Poland (approval No. 48/2012). Male, 7-week-old SHRs were divided into 2 groups: a study group on a supplemented diet (SHR/E group; $\mathrm{n}=8$ ) and a control group on a standard Labofeed B diet (SHR/C group; $n=8)$. The rats from the SHR/E group were given the supplemented diet for 12 weeks.

In our study, we used phospholipid fraction obtained from hen eggs and enriched naturally through feeding the hens substances such as PUFAs (DHA, for example); in this way, the yolk phospholipids were improved. The composition of the fraction was analyzed by gas chromatography/mass spectrometry (GC/MS). The egg phospholipid fraction is a mixture of phosphatidylcholine and phosphatidylethanolamine (the composition is detailed in Table 1). The diet of the study group after enrichment with phospholipid formulation contained $5 \%$ phosphatidylcholine, $0.65 \%$ omega- 3 fatty acid and $0.95 \%$ omega- 6 fatty acid, calculated globally.

Table 1. Composition [\%] of fatty acids in the phospholipid fraction of egg yolk

\begin{tabular}{|c|c|}
\hline Composition of supplement & {$[\%]$} \\
\hline PC & 81.72 \\
\hline$P E$ & 18.27 \\
\hline Fatty acid profile & {$[\%]$} \\
\hline$\omega-3$ & 10.82 \\
\hline$\omega-6$ & 15.79 \\
\hline$\omega 6 / \omega 3$ & 1.46 \\
\hline Saturated & 41.05 \\
\hline Unsaturated & 58.95 \\
\hline MUFA & 32.34 \\
\hline PUFA & 26.61 \\
\hline
\end{tabular}

PC - phosphatidylcholine; PE - phosphatidylethanolamine;

MUFA - monounsaturated fatty acid; PUFA - polyunsaturated fatty acid. 
Spontaneously hypertensive rats (age of 15 week) were fitted with DSI telemetry devices (PA11PC40, Data Sciences International, St. Paul, USA) at the $8^{\text {th }}$ week of diet supplementation. The animals were anesthetized with isoflurane (2-3\%) through inhalation with a flow rate of $1.5 \mathrm{~L} / \mathrm{min}$. For all animals, the isoflurane concentration was adjusted by decreasing the dose to the minimum which would provide analgesia. An aseptic laparotomy was performed to expose the abdominal aorta. A catheter tip was inserted into the aorta and secured with medical glue. The body of the telemetry device was left inside the abdominal cavity and secured to the abdominal wall during suture closure of the incision. The system was programmed to collect data on systolic, mean and diastolic pressure.

Following the $12^{\text {th }}$ week of study, blood pressure was measured. The blood pressure measurements were taken before the stress procedure under normal conditions in each animal for $12 \mathrm{~h}$. Subsequently, a model of acute stress with white noise was used. Blood pressure measurement was carried out during the exposure to stress. One 40-minute cycle of blood pressure monitoring in the presence of a stressor was carried out in each subject.

White noise was emitted at $100 \mathrm{~dB}$ through speakers located $30 \mathrm{~cm}$ above the cage. Blood pressure was measured simultaneously. The volume of sound was monitored by a decibel meter. After pressure measurement, the rats were anesthetized with isoflurane and sacrificed by cervical dislocation. Differences between the parameters under stress and before the procedure of acute stress $(\Delta)$ were calculated. Changes in the cardiovascular parameters $(\Delta)$ caused by acute stress were used as an indicator factor of stress level.

\section{Statistical analysis}

The data is expressed as mean \pm standard error of the mean (SEM) for each parameter and group. The difference between means for independent variables was analyzed using the Student's t-test or Mann-Whitney U test, while differences within one group (dependent variables) were analyzed using the dependent Student's t-test or Wilcoxon test. A p-value $<0.05$ was considered statistically significant. The statistical analysis was performed with STATISTICA v. 10.0 software (StatSoft Inc., Tulsa, USA).

\section{Results}

Twelve-hour measurement intervals under normal conditions showed that the mean pressure, systolic pressure and diastolic pressure were significantly lower in the SHRs fed an enriched diet than in the control group (159/106 mm Hg vs 174/117 mm Hg). In all groups, the acute stress exposure evoked a significant increase in mean pressure, systolic pressure, diastolic pressure, and heart rate, whereas pulse pressure significantly increased only in the control group (Table 2). Moreover, a stress-induced increase in mean pressure $(\Delta \mathrm{P})$ and systolic pressure $(\triangle \mathrm{SP})$ was significantly lower in the study group than in the control group ( $\triangle \mathrm{P}: 23.42 \mathrm{~mm} \mathrm{Hg}$ vs $36.86 \mathrm{~mm} \mathrm{Hg} ; \Delta \mathrm{SP}: 24.95 \mathrm{~mm} \mathrm{Hg}$ vs $40.02 \mathrm{~mm} \mathrm{Hg}$ ). The increase in these parameters caused by stress was less pronounced in rats fed a diet enriched with PUFAs. However, the difference in stress-induced diastolic pressure changes $(\triangle \mathrm{DP})$ between groups did not reach statistical significance $(\mathrm{p}=0.07)$ due to the small number of animals (Table 3$)$.

\section{Discussion}

There has been an increased focus on identifying the natural components of foods which could be used in the prevention and treatment of hypertension. Grains, vegetables, fruits, dairy products, meat, chicken, eggs, fish, soybean, tea, wine, mushrooms, and lactic acid bacteria are various natural food sources with potential antihypertensive effects. ${ }^{19}$ Blood pressure is regulated through complex mechanisms, including the modification of angiotensinconverting enzyme (ACE) activity and changes in oxidative

Table 3. Stress-induced increase of mean pressure $(\triangle P)$, systolic pressure $(\triangle S P)$, diastolic pressure $(\triangle D P)$, pulse pressure $(\triangle P P)$, and heart rate $(\triangle H R)$ in the control group (SHR/C) and study group (SHR/E)

\begin{tabular}{|l|c|c|}
\multicolumn{1}{|c|}{$\begin{array}{c}\text { Calculated } \\
\text { parameters }\end{array}$} & SHR/C & SHR/E \\
\hline$\triangle \mathrm{P}[\mathrm{mm} \mathrm{Hg}]$ & $36.86 \pm 3.73$ & $23.42 \pm 4.27^{*}$ \\
\hline$\triangle \mathrm{SP}[\mathrm{mm} \mathrm{Hg}]$ & $40.02 \pm 4.02$ & $24.95 \pm 4.23^{*}$ \\
\hline$\triangle \mathrm{DP}[\mathrm{mm} \mathrm{Hg}]$ & $34.43 \pm 3.72$ & $22.00 \pm 4.10^{* *}$ \\
\hline$\triangle \mathrm{PP}[\mathrm{mm} \mathrm{Hg}]$ & $5.58 \pm 1.16$ & $2.96 \pm 0.97$ \\
\hline$\triangle \mathrm{HR}[\mathrm{bpm}]$ & $99.71 \pm 20.10$ & $93.49 \pm 13.37$ \\
\hline
\end{tabular}

${ }^{*} \mathrm{p}<0.05$; statistically significant difference in comparison to SHR/C. ** $p=0.07$ in comparison to SHR/C. $\Delta$ - difference between parameter under stress and parameter before acute stress procedure. 
status. Antioxidants may decrease arterial blood pressure by decreasing oxidative stress, thus preserving the activity of nitric oxide synthase (NOS) and increasing nitric oxide bioavailability. It was previously demonstrated that egg consumption may decrease oxidative stress levels; thus, our results are in agreement with those reported by Jahandideh et al. ${ }^{20}$ Peptides obtained from egg white, such as novokinin, may act as a potent hypotensive factor through the AT(2) receptor. ${ }^{21}$ Fatty acids from fish oil are known to decrease blood pressure. ${ }^{22}$ It has been shown in clinical trials that n-3 PUFAs reduce the incidence of CVD and sudden cardiac death, and they decrease blood pressure. ${ }^{23}$ It has been demonstrated that dietary compounds rich in PUFAs reduce pressor responses of the mesenteric artery to norepinephrine. ${ }^{24}$ It is well-established that egg yolk contains peptides that demonstrate antioxidant, ACE-inhibitory and antidiabetic ( $\alpha$-glucosidase and DPP-IV inhibitory) activities. ${ }^{25}$

However, there has not been any clinical experience with the use of phospholipid fraction obtained from hen egg yolk as a mean to control blood pressure. Hen eggs are a relatively inexpensive source of not only fatty acids but also of amino acids and arginine, a precursor to nitric oxide. ${ }^{26}$ In our study, rats fed a diet enriched with phospholipid fraction obtained from egg yolk had decreased blood pressure compared to the control group. The decrease in blood pressure was even more significant in rats exposed to acute stress.

Stress has become a prevalent part of people's lifestyle; therefore, the effect of stress on blood pressure is of increasing relevance and importance. We used noise as a stressor because it is a constant component of modern life and is considered to be a hypertensive factor. For what is likely the first time, advanced cardiovascular telemetry devices were utilized to evaluate the response to stress. The use of a modern experimental model which allows blood pressure monitoring in conscious rats via telemetry seems to be an important step in identifying the effects of phospholipid fraction on stress and blood pressure. It is well-known that acute stress causes a rise in blood pressure and heart rate. This new method of stress level evaluation might be used in the pre-clinical selection of bioactive products with the highest anti-stress potential.

Our results showing the influence of PUFAs on blood pressure are in agreement with the data of Jayasooriya et al., who showed that PUFA supplementation reduces hypertension in rats (animals with high ANG II activity). ${ }^{27}$ Thus, our findings in this study expand on existing evidence that PUFAs decrease blood pressure. ${ }^{28}$ Moreover, we observed that a diet enriched with PUFAs reduces the increase in blood pressure evoked by acute stress. We observed that phospholipid fraction obtained from hen egg yolk rich in PUFAs acts as a hypotensive agent and that it could be used in the prevention or treatment of hypertension evoked or exacerbated by acute stress. This finding suggests that egg phospholipid fraction could play an important role in the prevention of blood pressure rise related to stress.
Moreover, if we suppose that blood pressure change is an indicator of stress level, this study would suggest that phospholipids demonstrate anti-stress activity. The use of a hypertensive animal model probably enhances this effect. However, further studies with different stressors and stress models are needed in order to confirm these early results. In the last decade, there has been increasing interest in foods that promote health and could prevent hypertension and CVD. Chicken eggs have been demonstrated to decrease blood pressure; therefore, they can be considered hypotensive agents because they may become a part of a daily diet as functional food.

\section{Conclusions}

The results of this study indicate that the phospholipid fraction rich in fatty acids which is obtained from egg yolk reduces the negative response of the cardiovascular system to acute stress in SHRs. Since the study demonstrated the beneficial effects of phospholipid fraction on blood pressure in rats exposed to stress, the implications for patients need to be tested in further studies.

\section{References}

1. Geleijnse JM, Giltay EJ, Grobbee DE, Donders ART, Kok FJ. Blood pressure response to fish oil supplementation: Metaregression analysis of randomized trials. J Hypertens. 2002;20(8):1493-1499.

2. Mozaffarian D, Geelen A, Brouwer IA, Geleijnse JM, Zock PL, Katan MB. Effect of fish oil on heart rate in humans: A meta-analysis of randomized controlled trials. Circulation. 2005;112(13):1945-1952.

3. Pauletto P, Puato $M$, Caroli MG, et al. Blood pressure and atherogenic lipoprotein profiles of fish-diet and vegetarian villagers in Tanzania: The Lugalawa study. Lancet. 1996;348(9030):784-788.

4. Morris MC, Sacks F, Rosner B. Does fish oil lower blood pressure? A meta-analysis of controlled trials. Circulation. 1993;88(2):523-533.

5. Schiano V, Laurenzano E, Brevetti G, et al. Omega-3 polyunsaturated fatty acid in peripheral arterial disease: Effect on lipid pattern, disease severity, inflammation profile, and endothelial function. Clin Nutr. 2008;27(2):241-247.

6. Kenny D, Warltier DC, Pleuss JA, Hoffmann RG, Goodfriend TL, Egan BM. Effect of omega-3 fatty acids on the vascular response to angiotensin in normotensive men. Am J Cardiol. 1992;70(15):1347-1352.

7. Nestel P, Shige H, Pomeroy S, Cehun M, Abbey M, Raederstorff D. The $n-3$ fatty acids eicosapentaenoic acid and docosahexaenoic acid increase systemic arterial compliance in humans. Am J Clin Nutr. 2002;76(2):326-330.

8. Kearney PM, Whelton M, Reynolds K, Muntner P, Whelton PK, He J. Global burden of hypertension: Analysis of worldwide data. Lancet. 2005;365(9455):217-223.

9. Wolf-Maier K, Cooper RS, Benegas JR, et al. Hypertension prevalence and blood pressure levels in 6 European countries, Canada and the United States. JAMA. 2003;289(18):2363-2369.

10. Castelli WP. Epidemiology of coronary heart disease: The Framingham Study. Am J Med. 1984;76(2A):4-12.

11. Lewington S, Clarke R, Qizilbash N, Peto R, Collins R; Prospective Studies Collaboration. Age-specific relevance of usual blood pressure to vascular mortality: A meta-analysis of individual data for one million adults in 61 prospective studies. Lancet. 2002;360(9349): 1903-1913.

12. Whelton PK. Epidemiology of hypertension. Lancet. 1994;344(8915): 101-106.

13. Wojtyla A, Bilinski $P$, Jaworska-Luczak B. Regulatory strategies to ensure food and feed safety in Poland: Update review. Ann Agric Environ Med. 20110;17:215-220. 
14. Isomaa $\mathrm{B}$, Almgren $\mathrm{P}$, Tuomi $\mathrm{T}$, et al. Cardiovascular morbidity and mortality associated with the metabolic syndrome. Diabetes Care. 2001;24(4):683-689.

15. Cuspidi C, Ambrosioni E, Mancia G, Pessina AC, Trimarco B, Zanchetti A APROS Investigators. Role of echocardiography and carotid ultrasonography in stratifying risk in patient with essential hypertension: The Assessment of Prognostic Risk Observational Survey. J Hypertens. 2002;20(7):1307-1314.

16. Pauletto P, Sarzani R, Rappelli A, Pessina AC, Sartore S. Vascular smooth muscle cell differentiation and growth response in hypertension. In: Laragh JH, Brenner BM, eds. Hypertension: Pathophysiology, Diagnosis and Management. $2^{\text {nd }}$ ed. New York, NY: Raven Press Publishers; 1995:697-709.

17. Grassi G, Mancia G. Hyperadrenergic and labile hypertension. In: Lip GH, Hall J, eds. Comprehensive Hypertension. Philadelphia, PA: Mosby Elsevier; 2007:719-726.

18. Grassi G, Cifkova R, Laurent S, et al. Blood pressure control and cardiovascular risk profile in hypertensive patient from Central and Eastern European countries: Results of BP-CARE study. Eur Heart J. 2011;32(2):218-225.

19. Huang WY, Davidge ST, Wu J. Bioactive natural constituents from food sources: Potential use in hypertension prevention and treatment. Crit Rev Food Sci Nutr. 2014;53(6):615-630.

20. Jahandideh F, Majumder K, Chakrabarti S, et al. Beneficial effects of simulated gastro-intestinal digests of fried egg and its fractions on blood pressure, plasma lipids and oxidative stress in spontaneously hypertensive rats. PLOS ONE. 2014;9(12):e115006.
21. Yamada Y, Yamauchi D, Yokoo M, Ohinata K, Usui H, Yoshikawa M. A potent hypotensive peptide, novokinin, induces relaxation by AT2and IP-receptor-dependent mechanism in the mesenteric artery from SHRs. Biosci Biotechnol Biochem. 2008;72(1):257-259.

22. Appel LJ, Miller ER, Seidler AJ, Whelton, PK. Does supplementation of diet with "fish oil" reduce blood pressure? A meta-analysis of controlled clinical trials. Arch Intern Med. 1993;153(12):1429-1438.

23. Calder PC. n-3 fatty acids and cardiovascular disease: Evidence explained and mechanisms explored. Clin Sci (Lond). 2004;107(1):1-11.

24. Skoczyńska A, Wojakowska A, Nowacki D, et al. Unsaturated fatty acids supplementation reduces blood lead level in rats. Biomed Res Int. 2015;2015:189190.

25. Zambrowicz A, Pokora M, Setner B, et al. Multifunctional peptides derived from an egg yolk protein hydrolysate: Isolation and characterization. Amino Acids. 2015;47(2):369-380.

26. Preli RB, Klein KP, Herrington DM. Vascular effects of dietary L-arginine supplementation. Atherosclerosis. 2002;162(1):1-15.

27. Jayasooriya AP, Begg DP, Chen N, et al. Omega-3 polyunsaturated fatty acid supplementation reduces hypertension in TGR(mRen-2)27 rats. Prostaglandins Leukot Essent Fatty Acids. 2008;78(1):67-72.

28. Nowacki D, Martynowicz H, Skoczyńska A, et. al. Lecithin derived from $\omega$-3 PUFA fortified eggs decreases blood pressure in spontaneously hypertensive rats. Sci Rep. 2017;7(1):12373. 